\title{
Renin-angiotensin system modulating treatments in the management of cardiovascular disease
}

As demand grows for more effective and evidence based management of cardiovascular disease, treatments which modify the renin-angiotensin system (RAS) have become a major focus for research. As a consequence, RAS modulating agents such as angiotensin converting enzyme (ACE) inhibitors and angiotensin II type $1\left(\mathrm{AT}_{1}\right)$ receptor blockers have attracted much scientific interest.

It is no surprise then that the current evidence for RAS modulating treatment - and the possible directions for the future-became the subject of a major international two day meeting, held in November 1999. While looking to the future, the meeting provided a valuable forum for assessing the current standard of cardiovascular evidence and practice, and reviewing its clinical implications.

The meeting explored the wealth of evidence that exists for ACE inhibitors across a wide range of cardiovascular indications, including post-myocardial infarction, in heart failure and in the renal and retinal complications of diabetes. In addition, the meeting examined the emerging role of $\mathrm{AT}_{1}$ receptor blockers, which have already been demonstrated in the management of hypertension, as well as the numerous studies currently underway to explore their benefits across the broader spectrum of cardiovascular disease.

The discussion included important new data reflecting the major themes of the meeting, including the first presentation to an international audience of STOPHypertension 2 (Swedish trial in old patients with hypertension 2), which showed that ACE inhibitors and calcium channel blockers are effective as standard treatment, and furthermore that ACE inhibitors demonstrated additional benefits. Also discussed in several presentations was the ATLAS (assessment of treatment with lisinopril and survival) study which clearly demonstrated the efficacy of high doses of the ACE inhibitor lisinopril in heart failure.

In addition the meeting explored the HOPE (heart outcomes prevention evaluation) study which adds to the considerable weight of evidence in favour of long acting, once daily ACE inhibitors in reducing the risks of cardiovascular events. This study showed that treatment with the ACE inhibitor ramipril in patients at high risk of cardiovascular events dramatically reduced the risk of cardiovascular death, non-fatal myocardial infarction, and stroke. Speculation as to whether this effect is related to ACE inhibitors in general is likely to continue; we may very well find that the positive effects of the ACE inhibitor seen in the HOPE study are shared by other long acting ACE inhibitors.

Discussions also extended to whether more complete blockade of the RAS - at the level of angiotensin converting enzyme and at receptor level—will yield additional benefits. This will be examined in future studies such as CALM (candesartan and lisinopril in microalbuminuria) which will examine the effect of this ACE and $\mathrm{AT}_{1}$ combination. In addition the CHARM (candesartan in heart failure: assessment of reduction in mortality and morbidity) study has an ACE and $\mathrm{AT}_{1}$ combination arm. Other directions were also examined, including data from the diabetic subgroup in HOPE which has demonstrated regression of coronary artery lesions in those treated with the ACE inhibitor. This could suggest a future role for statin/ACE inhibitor combinations.

The potential for RAS modulating treatments is evident, both on their own and in conjunction with, for example, glitazones or statins, and they provide exciting directions for future research. It is clear that the RAS and its relation with cardiovascular disease will continue to provide a focus for scientific and clinical research for many years to come.

It gives me great pleasure to recommend this supplement to you as an important contribution in this ongoing academic debate and as a comprehensive way to update your knowledge in this field.

LARS RYDÉN

Guest editor

Department of Cardiology,

Karolinska Hospital,

Stockholm,

Sweden

email:lars.ryden@ks.se 